Case Report

\title{
GERSTMANN SYNDROME IN A YOUNG MAN: A CASE REPORT
}

\author{
Iv. Dimitrov ${ }^{\text {* }}$, S. Atanasova ${ }^{2}$, A. Kaprelyan' ${ }^{2}$, B. Ivanov $^{3}$, V. Nestorova ${ }^{4}$, K. Drenska ${ }^{2}$, \\ Zh. Chuperkova ${ }^{5}$, Iv. Aleksandrov ${ }^{1}$ \\ ${ }^{1}$ Department of Nursing, Sliven Affiliate, Prof. Paraskev Stoyanov Medical University, Varna, \\ Bulgaria \\ ${ }^{2}$ Department of Neurology and Neuroscience, Faculty of Medicine, Prof. Paraskev Stoyanov Medical \\ University, Varna, Bulgaria \\ ${ }^{3}$ Department of Clinical Medical Sciences, Faculty of Dental Medicine, Prof. Paraskev Stoyanov \\ Medical University, Varna, Bulgaria \\ ${ }^{4}$ Department of Physiotherapy, Rehabilitation, Thalassotherapy, Occupational Diseases, Faculty of \\ Public Health, Prof. Paraskev Stoyanov Medical University, Varna, Bulgaria \\ ${ }^{5}$ Child Neurology Sector, Second Pediatric Clinic, MHAT St. Marina, Varna, Bulgaria
}

\begin{abstract}
Gerstmann syndrome is a classical cerebral syndrome in neurology, named after Joseph Gerstmann, a Jewish Austrian-born American neurologist. Patients present with a tetrad of cognitive symptoms, including agraphia, acalculia, finger agnosia and left-right disorientation. The syndrome is known to result from a lesion of the posterior portion of the dominant parietal lobe and is usually due to stroke or to developmental problems. We describe the case of a 35-year-old man whose illness debuted about 9 months before the initial presentation to the neurology clinic, with memory complaints, anxiety, verbal aggression, sleeping problems, as well as subjective word finding difficulty and depressed mood. The patient had 3 out of the 4 classic symptoms of Gerstmann syndrome, among other, mostly neuropsychiatric symptoms. Initially, structural lesions were sought for, but were not found on magnetic resonance imaging. Psychiatric conditions were discussed but not confirmed by the consulting psychiatrist. We are prone to accepting a non-organic reason for the condition of the patient, but follow-up of the clinical course and repeated assessments, including neuropsychological and psychiatric evaluations, structural and possibly functional neuroimaging will be required to verify and confirm this presumption.
\end{abstract}

Key words: Gerstmann syndrome, agraphia, acalculia, finger agnosia, left-right disorientation

\section{INTRODUCTION}

Gerstmann syndrome is a classical cerebral syndrome in neurology. It was named after Joseph Gerstmann, a Jewish Austrian-born American neurologist (1). Patients present with a tetrad of cognitive symptoms, including agraphia, acalculia, finger agnosia and leftright disorientation (2). The syndrome is

\footnotetext{
*Correspondence to: Ivan Dimitrov, Department of Nursing, Sliven Affiliate, Prof. Paraskev Stoyanov Medical University, Sveta Marina University Hospital, First Clinic of Neurology, 1 Hristo Smirnenski st., 9010 Varna, Bulgaria, Telephone: +35952978 524, E-mail: ivan.dimitrov@mu-varna.bg
}

known to result from a lesion of the posterior portion of the dominant parietal lobe (3). The affected area is usually the angular gyrus (4) which is responsible for processing the visual images of letters and words in meaningful information, as is done during reading. Such lesions in adults are usually seen in stroke, while in children the syndrome can be related to developmental problems (Developmental Gerstmann syndrome). In the latter cases the aetiology remains unknown (5). The clinical manifestations that constitute the syndrome are heterogeneous. That is why some authors consider unlikely the possibility that damage to the same population of neurons should account for all of the four symptoms, and explain the 
syndrome with lesions causing disconnection of co-localized tracts in the subcortical white matter of the parietal lobe (6).

\section{CASE REPORT}

We describe the case of a 35-year-old man whose illness debuted about 9 months before the initial presentation to the neurology clinic, with memory complaints, anxiety, verbal aggression, sleeping problems, as well as subjective word finding difficulty. According to his wife, he had become depressed in the recent months and had developed the habit to ask the same questions multiple times. For the last month he had had 2 collapses with measured close values of systolic and diastolic blood pressure, with no convulsions. Ten years earlier, he had suffered a head trauma whiplash injury with no fractures or loss of consciousness. The patient had no other relevant medical history. He denied alcohol or other substance abuse.

In the outpatient care, due to the described complaints, he was consulted by a neurologist and a psychiatrist, electroencephalography and carotid/vertebral Doppler ultrasonography were performed, which were normal. Neuropsychological assessment showed mild to moderate frontal dysfunction, impaired attention, mild disturbance of immediate recall and moderate of delayed recall, emotional and behavioral disturbances. Mini- Mental State Exam (MMSE) score was 29 points. The patient was classified as "neurasthenic" and treatment with valproate and hydroxyzine
DIMITROVIV., et al. hydrochloride in usual doses was prescribed. He had not carried out the treatment, stating "personal reasons".

As the symptoms persisted, the patient was sent to the neurology clinic for admission, further assessment and treatment. The neurological exam was normal. Only elevated ALAT (77.0 U/L) was found on laboratory assessment. Abdominal ultrasonography showed no pathological changes. Neuropsychological assessment was repeated. MMSE score was 27 points with evidence of dyscalculia. Isaacs set test showed normal category verbal fluency with a score of 36 points. Clock drawing test was normal, with a maximal score of 10 points according to Sunderland's scoring method. Luria's memory test showed fixation of $66 \%$ (lower normal level) and reproduction of $75 \%$ (middle normal level). Finger agnosia and left-right disorientation were present. Psycho-emotional instability and fluctuations of cognitive processing were noted.

The consultant psychiatrist reported that the patient was calm, orientated, euthymic, with mild alterations of thinking, but no psychiatric illness was diagnosed. Brain magnetic resonance imaging (MRI) was performed in order to seek for structural lesions, but no pathology was detected (Figure 1). The patient was discharged in good general condition, with recommendations for neurologic, psychiatric, neuropsychological and, if needed, neuroimaging follow-up.

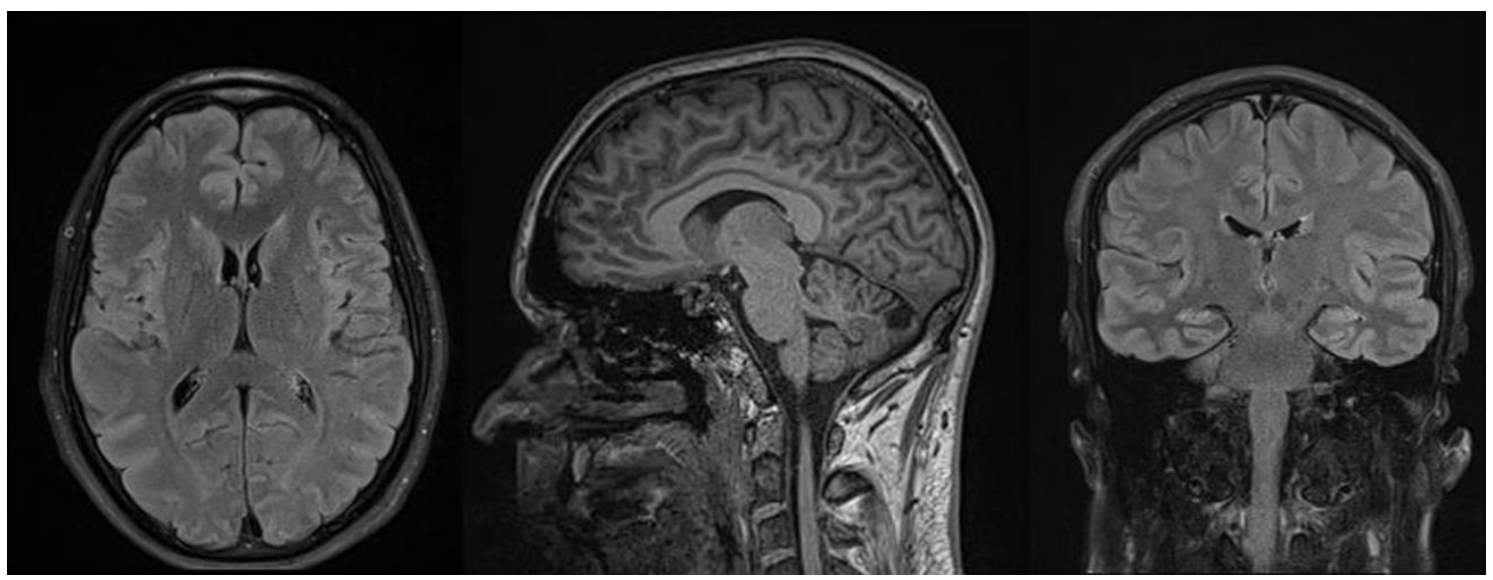

Figure 1. Normal MRI of the brain in axial, sagittal and coronal planes

\section{DISCUSSION}

The described case shows the presence of 3 out of the 4 classic symptoms of Gerstmann syndrome: dyscalculia, finger agnosia and leftright disorientation, among other, mostly neuropsychiatric symptoms. Initially, structural lesions were sought for, but were not found on MRI. The patient was young and had no vascular risk factors, which additionally reduced the likelihood of vascular disease to be the reason for the clinical manifestations. In line with the statement that Gerstmann syndrome may result from intraparietal disconnection after damage to a focal region of subcortical white matter $(7,8)$, and taking into account the age of the patient, we also 
considered a demyelinating disease, which, again, was not confirmed by MRI. The history of whiplash injury was taken into account, but evidences for connection with the current condition were not convincing. As psychiatric/neuropsychiatric symptoms were dominating the clinical picture, a psychiatric disease was an important differential diagnostic option. For instance, it has been stated in the literature that the symptoms of Gerstmann syndrome can be present in schizophrenia patients, even in the early stages (9). Some mild alterations of thinking were noted in our patient, but the consultant psychiatrist did not diagnose a psychiatric condition. Nevertheless, in this case we are prone to accepting a non-organic reason for the condition of the patient. Malingering was discussed, but considered unlikely. Follow-up of the clinical course and repeated assessments, including neuropsychological and psychiatric evaluations, structural and possibly functional neuroimaging will be required to verify and confirm this presumption.

\section{ACKNOWLEDGEMENT}

No funding was obtained for this study.

\section{REFERENCES}

1. Triarhou, LC. Josef Gerstmann (18871969). J Neurol. Apr 2008;255(4):614-615.

2. Roeltgen, DP, Sevush, S, Heilman, KM. Pure Gerstmann's syndrome from a focal
DIMITROV IV., et al. lesion. Archives of Neurology, 40(1):46-47, 1983.

3. Wilkins, RH and Brody, IA. Gerstmann's syndrome. Archives of Neurology, 24(5):475-475, 1971.

4. João, RB, Filgueiras, RM, Mussi, ML, Barros, JEFd. Transient Gerstmann syndrome as manifestation of stroke: Case report and brief literature review. Dementia \& Neuropsychologia, 11:202-205, 2017.

5. Kinsbourne, MM, Warrington, EK. The developmental Gerstmann syndrome. Archives of Neurology, 8(5):490-501, 1963.

6. Rusconi, E, Pinel, P, Dehaene, S, Kleinschmidt, A. The enigma of Gerstmann's syndrome revisited: a telling tale of the vicissitudes of neuropsychology. Brain, 133(2):320-332, 2010.

7. Rusconi, E, Pinel, P, Eger, E, et al. A disconnection account of Gerstmann syndrome: functional neuroanatomy evidence. Ann Neurol, 66(5):654-662, 2009.

8. Rusconi, E. Gerstmann syndrome: historic and current perspectives. Handb Clin Neurol, 151:395-411, 2018.

9. Liu, X, Zhuo, C, Qin, W, et al. Selective functional connectivity abnormality of the transition zone of the inferior parietal lobule in schizophrenia. NeuroImage: Clinical, 11:789-795, 2016. 